\title{
Critères de choix et de réforme des reproducteurs mâles et femelles dans les élevages de porcs des départements de l'Ouémé et du Plateau au Bénin
}

\author{
Ignace Ogoudanan Dotché ${ }^{1 *}$ Serge Gbênagnon Ahounou ${ }^{1}$ \\ Chakirath Folakè Arikè Salifou ${ }^{1}$ Rodrigue Biobou ${ }^{1}$ Pascal \\ Sègbégnon Kiki ${ }^{1}$ Benoit Govoeyi ${ }^{1,2}$ Nicolas Antoine- \\ Moussiaux ${ }^{2}$ Jean-Paul Dehoux ${ }^{3}$ Guy Apollinaire Mensah ${ }^{4}$ \\ Souaïbou Farougou ${ }^{1}$ Pierre Thilmant ${ }^{2,5}$ Issaka Youssao \\ Abdou Karim ${ }^{1}$ Benoît Koutinhouin ${ }^{1}$
}

Mots-clés

Porcin, conduite d'élevage, verrat, truie, performance de reproduction, réforme des animaux, Bénin

Submitted: 13 July 2017

Accepted: 3 July 2018

Published: 9 September 2018

DOI: $10.19182 /$ remvt.31224

\section{Résumé}

L'objectif de l'étude a été de décrire les critères de choix et de réforme des reproducteurs porcins au Bénin. Une enquête a été réalisée auprès de 65 éleveurs porcins. L'analyse factorielle des correspondances a été utilisée pour identifier des groupes d'éleveurs en fonction de leurs pratiques au niveau de chaque sexe. Le groupe 1 était composé en majorité de personnes non scolarisées élevant principalement des porcs locaux en liberté. Le groupe 2 était composé majoritairement d'éleveurs de niveau du primaire ou du secondaire. Le groupe 3 comprenait des éleveurs non scolarisés et de niveau du secondaire. Les éleveurs des groupes 2 et 3 utilisaient des races améliorées et des animaux croisés, en claustration. Les critères les plus utilisés pour le choix des mâles dans les élevages ont été l'état de santé, la conformation et les testicules très développés, le nombre de tétines, l'absence de défaut génétique, et la croissance. L'absence de défaut génétique a été plus utilisée dans les groupes 2 et 3 , puis les testicules développés dans le groupe 1. Les critères les plus utilisés pour le choix des femelles dans les trois groupes ont été l'état de santé, la taille de la portée, les liens de parenté et la croissance des porcelets. La plupart de ces critères ont été moins utilisés dans le groupe 1. Les critères les plus utilisés pour la réforme des verrats ont été l'âge et la perte de libido. Les principaux critères de réforme des femelles âgées dans tous les groupes ont été le rang de portée et la baisse de la taille de la portée. Cette dernière a été plus utilisée dans les groupes 2 et 3 . Pour conclure, les éleveurs disposaient de bons critères de choix et de réforme des reproducteurs qu'il faudrait améliorer afin d'augmenter leur productivité.

- Pour citer cet article : Dotché I.O., Ahounou S.G., Salifou C.F.A., Biobou R., Kiki P.S., Govoeyi B., AntoineMoussiaux N., Dehoux J.-P., Mensah G.A., Farougou S., Thilmant P., Youssao Abdou Karim I., Koutinhouin B., 2018. Selection and culling criteria for breeding boars and sows in pig farms from Oueme and Plateau departments in Benin. Rev. Elev. Med. Vet. Pays Trop., 71 (1-2): 47-57, doi: 10.19182/remvt.31224

\footnotetext{
1. Laboratoire de biotechnologie animale et de technologie des viandes, Département de production et santé animales, Ecole polytechnique d'AbomeyCalavi, Université d'Abomey-Calavi, 01 BP 2009, Cotonou, Bénin.

2. Département de production animale, Faculté de médecine vétérinaire, Université de Liège, B-4000 Liège, Belgique.

3. Unité de chirurgie expérimentale, Faculté de médecine, Université catholique de Louvain, 1200 Bruxelles, Belgique.

4. Institut national de recherches agricoles du Bénin (INRAB), Cotonou 01, Bénin. 5. Centre provincial liégeois de productions animales (CPL Animal), Argenteau, Belgique.

* Auteur pour la correspondance

Tél. : +229675652 20

Email : ogoudanan@yahoo.fr / dotcheign@gmail.com
}

\section{INTRODUCTION}

Le porc est l'une des espèces les plus utilisées au Bénin dans les productions animales. Son élevage est plus développé au sud et surtout dans les départements de l'Ouémé et du Plateau qui détiennent 27,5\% du cheptel national porcin (Agbokounou et al., 2016a ; Countrystat, 2017). Les races utilisées dans les élevages porcins de cette zone sont très diverses. Il s'agit de la race locale, des races améliorées pures représentées surtout par le Large White et le Landrace, puis les animaux croisés entre ces différents types génétiques (Djimènou et al., 2017). Ces animaux sont élevés dans les systèmes traditionnels dominés par l'élevage en plein air et un apport limité en intrants, ou dans des systèmes améliorés caractérisés par la claustration des animaux et un investissement élevé de l'éleveur (Agbokounou et al., 
2017b). La production issue de ces différents modes de production est faible et ne permet pas de satisfaire les besoins des consommateurs. Des efforts ont été faits pour l'amélioration de la productivité pondérale par l'alimentation et la génétique. Ainsi, de nombreuses formules alimentaires ont été conçues à partir de matières premières locales et d'aliments non conventionnels pour améliorer la productivité des animaux (Accodji et al., 2009 ; Hedji et al., 2015). Pour ce qui concerne la génétique, les performances de croissance des porcelets ont été améliorées par croisement avec les races exotiques, et les descendants F1 issus de ces croisements ont des performances de reproduction supérieures à celles des porcs locaux (Agbokounou et al., 2016a). Des essais ont été également faits pour améliorer la productivité numérique par l'amélioration du mode d'élevage (Agbokounou et al., 2016a). Les résultats de ces travaux auraient pu être plus performants si les reproducteurs mâles et femelles avaient été choisis sur la base de critères de sélection objectifs et si la réforme s'était faite au moment opportun. Le choix des animaux reproducteurs est très important, car il permet à l'éleveur de sélectionner les animaux en fonction de ses objectifs de production, de réaliser du progrès génétique, et de prévenir les effets de la consanguinité sur les performances zootechniques et sur la santé des animaux. Il permet également de limiter les temps improductifs des animaux et de maintenir une bonne démographie et des performances stables. Par conséquent, l'identification du moment idéal de réforme des reproducteurs en élevage porcin permet d'éviter des pertes économiques pour l'éleveur.

La présente étude a eu pour but de répertorier les critères de choix et de réforme des reproducteurs mâles et femelles dans les élevages porcins du Bénin. Les résultats issus de cette étude pourront permettre d'améliorer la gestion de la reproduction afin d'envisager l'introduction des biotechnologies de reproduction et principalement l'insémination artificielle dans les élevages du Sud-Bénin.

\section{MATERIEL ET METHODES}

\section{Cadre d'étude}

L'étude a été réalisée dans les départements de l'Ouémé et du Plateau au sud du Bénin. Le département de l'Ouémé se trouve entre $6^{\circ} 40^{\prime}$ $\mathrm{N}$ et $2^{\circ} 30^{\prime} \mathrm{E}$ et couvre une superficie de 1281 kilomètres carrés avec une population de 1100404 habitants en 2013 (INSAE, 2015). Les travaux ont été conduits dans les communes d'Adjarra, AkproMissérété, Avrankou, Porto-Novo et Sèmè-Kpodji (figure 1) de ce département.

Le département du Plateau est compris entre $7^{\circ} 10^{\prime} \mathrm{N}$ et $2^{\circ} 34^{\prime} \mathrm{E}$ et couvre une superficie de 3264 kilomètres carrés, soit environ $3 \%$ du territoire national pour une population totale de 622372 habitants (INSAE, 2015). Dans ce département, les travaux ont été conduits dans les communes d'Adja-Ouèrè, Ifangni, Pobè et Sakété (figure 1).

La méthodologie utilisée pour la collecte des données a été celle de l'enquête rétrospective par entretien avec l'éleveur. Cette enquête a permis de recueillir aussi bien les informations sur l'éleveur que sur la gestion de la reproduction dans son exploitation. Les éleveurs ont été choisis suivant les critères d'accessibilité et leur disponibilité à fournir les informations. La collecte des données a eu lieu dans 65 élevages des départements de l'Ouémé et du Plateau du 18 juillet au 14 octobre 2016. Une fiche d'enquête à choix multiple a été utilisée. Les critères de choix et de réforme contenus dans la fiche d'enquête ont été recueillis auprès des éleveurs lors d'une préenquête. Les questions étaient fermées et portaient sur : l'identification et la formation des éleveurs (non scolarisé, niveau primaire, niveau secondaire et autres), le système de production (élevage en plein air, élevage en claustration totale ou temporaire), les types génétiques utilisés (race locale, race améliorée ou animaux croisés), les performances zootechniques, les critères de choix des verrats et des cochettes, et les critères de réforme des verrats et des truies. Les données sur les variables quantitatives comme l'âge d'entrée en reproduction, l'âge au sevrage, l'âge à la réforme, l'âge à la castration, l'intervalle sevrage-saillie, la taille de la portée et le nombre de tétines ont été recueillies sur déclaration des éleveurs. Le thème race améliorée a été utilisé pour désigner les races exotiques et les produits de divers croisements réalisés entre ces races. Les croisés étaient les produits de croisement entre la race améliorée et la race locale.

\section{Analyses statistiques}

Après l'encodage, les données ont été analysées avec le logiciel SAS (2013). Au total, 60 variables ont été retenues pour les analyses. La procédure Proc corresp a été utilisée pour l'analyse factorielle des correspondances (AFC). Les variables prises en compte ont été : le niveau d'étude, le mode d'élevage, la race utilisée, les critères de choix et de réforme des reproducteurs. Une classification ascendante hiérarchique sur la base des caractéristiques des élevages sur les composantes de l'AFC les plus significatives a ensuite été réalisée. Des groupes d'éleveurs ont ainsi été identifiés et caractérisés.

Pour les variables quantitatives (taille du cheptel, âge à l'entrée en reproduction, âge au sevrage, âge à la réforme, âge à la castration, taille de la portée, rang de mise bas, nombre de tétines et durée sevrage-accouplement), une analyse de variance a été réalisée par la procédure Proc GLM. Le seul facteur de variation considéré dans le modèle d'analyse de variance a été l'effet du groupe d'élevage. Le test F de Fisher a été utilisé pour déterminer la significativité de l'effet
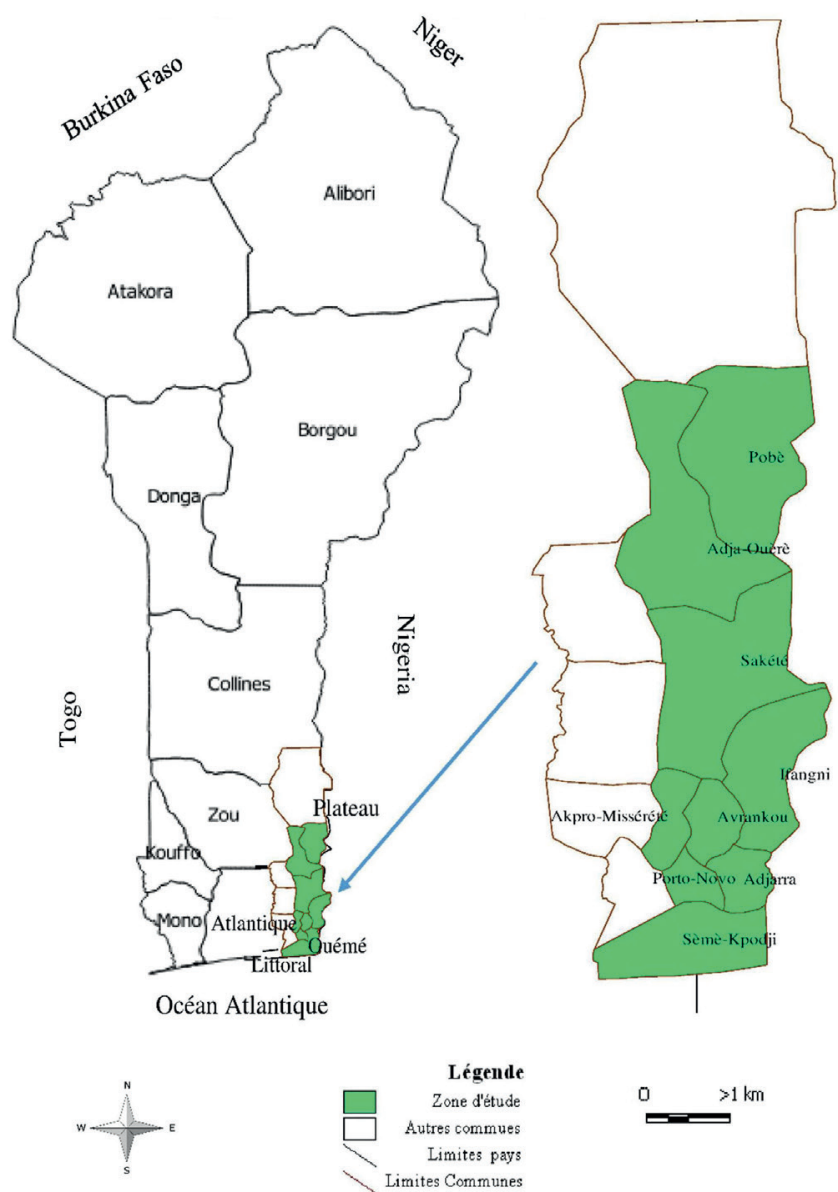

Figure 1 : zone d'étude sur les critères de sélection et de réforme des porcs au Sud-Bénin. 
groupe d'élevage et les comparaisons entre les moyennes de chacune des variables par groupe d'élevage ont été faites deux à deux avec le test $t$ de Student.

Pour les variables qualitatives (niveau d'étude, races élevées, mode d'élevage, critères de choix, et critères de réforme des reproducteurs mâles et femelles), les fréquences observées ont été calculées par la procédure Proc FREQ. L'effet du groupe sur les fréquences a été mis en évidence par le test du chi carré, et la comparaison des fréquences relatives entre groupes deux à deux a été faite par le test bilatéral de Z. Pour chaque fréquence relative, un intervalle de confiance $(I C)$ à $95 \%$ a été calculé selon la formule :

$$
I C=P \pm 1,96 \sqrt{\frac{P(1-P)}{N}}
$$

où $P$ est la fréquence relative et $N$ la taille de l'échantillon.

\section{RESULTATS}

\section{Profil des élevages enquêtés}

Trois axes ont été retenus pour l'interprétation des résultats de l'analyse factorielle des correspondances $\left(\chi^{2}=358,8\right)$ pour les critères de choix et de réforme des verrats. Chaque axe correspondait à un groupe d'éleveurs et chaque groupe correspondait à un type d'éleveur. La contribution à l'inertie totale des trois axes factoriels a été de $48,1 \%$ (24,1 \% pour l'axe $1,13,3 \%$ pour l'axe 2 et $10,6 \%$ pour l'axe 3). Le groupe 1 était composé de 11 éleveurs et les groupes 2 et 3 de 27 éleveurs chacun. Parmi les éleveurs formant le groupe 1, $72,7 \%$ étaient du Plateau et $27,3 \%$ de l'Ouémé. Le groupe 2 regroupait $51,8 \%$ d'éleveurs de l'Ouémé et 48,1\% du Plateau, et le groupe 3 comprenait 59,3\% d'éleveurs du Plateau et 40,7\% de l'Ouémé.

Trois axes ont été retenus également pour l'interprétation des résultats de l'analyse factorielle des correspondances $\left(\chi^{2}=370,7\right)$ pour les critères de choix des cochettes et de réforme des truies. Chaque axe correspondait à un groupe d'éleveurs et chaque groupe correspondait à un type d'éleveur. La contribution à l'inertie totale des trois axes factoriels a été de $46,2 \%$ (27,1 \% pour l'axe $1,11 \%$ pour l'axe 2 et $8 \%$ pour l'axe 3 ). Les groupes 1,2 et 3 étaient composés respectivement de 13 (92,3 \% du Plateau et 7,7 \% de l'Ouémé), 26 (46,2\% du Plateau et 53,8 \% de l'Ouémé) et 23 (56,5\% du Plateau et 43,5\% de l'Ouémé) éleveurs.

Les modes d'élevage pratiqués par l'ensemble des éleveurs enquêtés étaient l'élevage en claustration totale (78,5\%), l'élevage en claustration temporaire $(13,8 \%)$ et l'élevage en liberté $(9,2 \%)$. L'élevage en liberté se pratiquait uniquement dans le groupe 1 (tableaux I et II). Ainsi, 54,5\% des éleveurs de ce groupe le pratiquaient selon les critères de choix et de réforme des mâles, et aucun éleveur ne le pratiquait dans les groupes 2 et 3 ( $p<0,001)$. Le même constat a été fait au niveau des critères de choix et de réforme des femelles mais la fréquence observée a été de $46,2 \%$ dans le groupe 1 . En revanche, l'élevage en claustration totale a été davantage pratiqué $(p<0,001)$ dans les groupes 2 et 3 que dans le groupe 1 (tableaux I et II). Les proportions d'éleveurs pratiquant l'élevage en claustration temporaire n'a pas varié d'un groupe à l'autre. Dans ce mode, les animaux étaient gardés dans les porcheries en saison de culture pour éviter les dégâts aux cultures, ou à l'âge adulte pour éviter les cas de vol. Il n'y avait pas d'éleveurs sélectionneurs. Le renouvellement des reproducteurs se faisait surtout par autorenouvellement. Toutefois, certains éleveurs se procuraient des reproducteurs chez des voisins. Le taux de renouvellement a été de $42 \%$ dans les exploitations et n'a pas varié d'un groupe à l'autre.

Les éleveurs rencontrés étaient des personnes non scolarisées ou ayant un niveau primaire et secondaire. Les personnes non scolarisées ont été plus souvent $(\mathrm{p}<0,001)$ présentes dans les groupes 1 et 3 que dans le groupe 2 pour les critères de choix et de réforme des mâles. Pour les critères de choix des femelles, ces personnes ont été plus souvent rencontrées $(\mathrm{p}<0,001)$ dans le groupe $1(84,6 \%)$ que dans les groupes $2(11,5 \%)$ et $3(26,1 \%)$. La proportion d'éleveurs ayant le niveau primaire dans le groupe 2 a été significativement plus

\section{Tableau I}

Différences entre groupes d'éleveurs de porcins identifiés par l'analyse factorielle des correspondances des critères de choix et de réforme des mâles au Sud-Bénin

\begin{tabular}{|c|c|c|c|c|c|c|c|c|}
\hline \multirow[t]{2}{*}{ Variables } & \multirow[t]{2}{*}{$\%$ général $(n=65)$} & \multicolumn{2}{|c|}{ Groupe 1 (n = 11) } & \multicolumn{2}{|c|}{ Groupe $2(n=27)$} & \multicolumn{2}{|c|}{ Groupe $3(n=27)$} & \multirow{2}{*}{$\begin{array}{l}\text { Ecart entre groupes } \\
\text { (test du } \chi^{2} \text { ) }\end{array}$} \\
\hline & & $\%$ & IC & $\%$ & IC & $\%$ & IC & \\
\hline \multicolumn{9}{|l|}{ Niveau d'étude } \\
\hline Non scolarisé & 36,9 & $72,7^{a}$ & {$[46,4-99]$} & $11,1^{b}$ & {$[-0,7-22,9]$} & $43,1^{\mathrm{a}}$ & {$[24,9-61,3]$} & $* * *$ \\
\hline Niveau primaire & 23,1 & $0,0^{b}$ & [0-0] & $44,4^{\mathrm{a}}$ & {$[25,7-63,1]$} & $11,1^{\mathrm{b}}$ & {$[-0,7-22,9]$} & $* *$ \\
\hline Niveau secondaire & 40,0 & $27,3^{\mathrm{a}}$ & {$[1-53,6]$} & $44,4^{\mathrm{a}}$ & {$[25,7-63,1]$} & $45,7^{\mathrm{a}}$ & {$[27,2-64,2]$} & NS \\
\hline \multicolumn{9}{|l|}{ Race des reproducteurs } \\
\hline Locale & 33,8 & $72,7^{\mathrm{a}}$ & {$[46,4-99]$} & $22,2^{b}$ & {$[6,5-37,9]$} & $29,6^{b}$ & {$[12,4-46,8]$} & $* *$ \\
\hline Améliorée & 47,7 & $27,3^{\mathrm{a}}$ & {$[1-53,6]$} & $48,1^{\mathrm{a}}$ & {$[29,3-66,9]$} & $55,6^{\mathrm{a}}$ & {$[36,9-74,3]$} & NS \\
\hline Croisée & 35,4 & $0,0^{b}$ & {$[0-0]$} & $44,4^{\mathrm{a}}$ & {$[25,7-63,1]$} & $40,7^{\mathrm{a}}$ & {$[22,2-59,2]$} & $*$ \\
\hline \multicolumn{9}{|l|}{ Mode d'élevage } \\
\hline Liberté & 9,2 & $54,5^{\mathrm{a}}$ & {$[25,1-83,9]$} & $0,0^{b}$ & {$[0-0]$} & $0^{b}$ & {$[0-0]$} & $* * *$ \\
\hline Claustration & 78,5 & $27,3^{b}$ & {$[1-53,6]$} & $92,6^{\mathrm{a}}$ & {$[82,7-102,5]$} & $85,2^{\mathrm{a}}$ & {$[71,8-98,6]$} & $* * *$ \\
\hline Claustration temporaire & 13,8 & $18,2^{\mathrm{a}}$ & {$[-4,6-41]$} & $7,4^{\mathrm{a}}$ & {$[-2,5-17,3]$} & $14,8^{\mathrm{a}}$ & {$[1,4-28,2]$} & NS \\
\hline
\end{tabular}

${ }^{*} \mathrm{p}<0,05 ;{ }^{* *} \mathrm{p}<0,01 ;{ }^{* * *} \mathrm{p}<0,001 ; \mathrm{NS}$ : non significatif ; IC : intervalle de confiance

$a, b$ Les pourcentages sur une même ligne suivis de la même lettre ne diffèrent pas significativement au seuil de $5 \%$. 


\section{Tableau II}

Différences entre groupes d'éleveurs de porcins identifiés par l'analyse factorielle des correspondances des critères de choix et de réforme des femelles au Sud-Bénin

\begin{tabular}{|c|c|c|c|c|c|c|c|c|}
\hline \multirow[t]{2}{*}{ Variables } & \multirow[t]{2}{*}{ \% général $(n=62)$} & \multicolumn{2}{|c|}{ Groupe $1(n=13)$} & \multicolumn{2}{|c|}{ Groupe $2(n=26)$} & \multicolumn{2}{|c|}{ Groupe $3(n=23)$} & \multirow{2}{*}{$\begin{array}{l}\text { Ecart entre groupes } \\
\left(\text { test du } \chi^{2}\right)\end{array}$} \\
\hline & & $\%$ & IC & $\%$ & IC & $\%$ & IC & \\
\hline \multicolumn{9}{|l|}{ Niveau d'étude } \\
\hline Non scolarisé & 32,3 & $84,6^{\mathrm{a}}$ & {$[65-104,2]$} & $11,5^{b}$ & {$[-0,8-23,8]$} & $26,1^{b}$ & {$[8,2-44,0]$} & $* * *$ \\
\hline Niveau primaire & 22,6 & $0^{\mathrm{b}}$ & {$[0,0-0,0]$} & $46,2^{\mathrm{a}}$ & {$[27,0-65,4]$} & $8,7^{b}$ & {$[-2,8-20,2]$} & $* * *$ \\
\hline Niveau secondaire & 45,1 & $15,4^{b}$ & {$[-4,2-35,0]$} & $42,3^{a, b}$ & {$[23,3-61,3]$} & $65,2^{\mathrm{a}}$ & {$[45,7-84,7]$} & $*$ \\
\hline \multicolumn{9}{|l|}{ Race des reproducteurs } \\
\hline Locale & 35,5 & $84,6^{\mathrm{a}}$ & {$[65-104,2]$} & $19,2^{b}$ & {$[4,1-34,3]$} & $26,1^{b}$ & {$[8,2-44,0]$} & $* * *$ \\
\hline Améliorée & 45,2 & $7,7^{b}$ & {$[-6,8-22,2]$} & $50,0^{\mathrm{a}}$ & {$[30,8-69,2]$} & $60,9^{a}$ & {$[41,0-80,8]$} & $* *$ \\
\hline Croisée & 37,1 & $7,7^{b}$ & {$[-6,8-22,2]$} & $46,1^{\mathrm{a}}$ & {$[26,9-65,3]$} & $43,4^{\mathrm{a}}$ & {$[23,1-63,7]$} & $*$ \\
\hline \multicolumn{9}{|l|}{ Mode d'élevage } \\
\hline Liberté & 9,7 & $46,2^{\mathrm{a}}$ & {$[19,1-73,3]$} & $0,0^{b}$ & {$[0,0-0,0]$} & $0,0^{b}$ & {$[0,0-0,0]$} & $* * *$ \\
\hline Claustration & 77,4 & $30,8^{b}$ & {$[5,7-55,9]$} & $88,5^{\mathrm{a}}$ & {$[76,2-100,8]$} & $91,3^{\mathrm{a}}$ & {$[79,8-102,8]$} & $* * *$ \\
\hline Claustration temporaire & 14,5 & $30,8^{a}$ & {$[5,7-55,9]$} & $11,5^{\mathrm{a}}$ & {$[-0,8-23,8]$} & $8,7^{\mathrm{a}}$ & {$[-2,8-20,2]$} & NS \\
\hline
\end{tabular}

${ }^{*} \mathrm{p}<0,05 ;{ }^{* *} \mathrm{p}<0,01 ;{ }^{* * *} \mathrm{p}<0,001 ; \mathrm{NS}$ : non significatif ; IC : intervalle de confiance

$\mathrm{a}, \mathrm{b}$ Les pourcentages sur une même ligne suivis de la même lettre ne diffèrent pas significativement au seuil de $5 \%$.

élevée que celles des groupes 1 et 3 pour les critères de choix et de réforme des mâles. Le même constat a été fait pour les critères de choix et de réforme des femelles. La proportion de personnes ayant le niveau secondaire n'a pas varié d'un groupe à l'autre pour les critères de choix et de réforme des mâles. En revanche, pour les critères de choix et de réforme des femelles, ces personnes ont été plus nombreuses dans le groupe 3 que dans le groupe 1 (tableau II).

Concernant les races, les porcs locaux étaient plus présents dans le groupe 1 que dans les deux autres groupes indépendamment du sexe. Certains éleveurs qui utilisaient les porcs croisés possédaient les trois types génétiques. Les porcs locaux étaient élevés par les personnes non scolarisées (figures 2 et 3). Les porcs croisés étaient élevés seulement par les éleveurs des groupes $2(44,4 \%)$ et $3(40,7 \%)$ pour les critères de choix et de réforme des mâles ; ils étaient plus présents dans les groupes 2 et 3 que dans le groupe 1 pour les critères de choix et de réforme des femelles. Les porcs améliorés étaient davantage utilisés dans les groupes 2 et 3 que dans le groupe 1 (tableau II).

L'alimentation des animaux était assurée par des mélanges de matières premières, et les résidus de récolte et de cuisine. Les matières premières utilisées étaient les tourteaux d'oléagineux, les sons de céréales, les sons d'oléagineux et les drêches industrielles.

Figure 2 : critères de choix et de réforme des mâles dans les départements de l'Ouémé et du Plateau au Sud-Bénin. Nscol : non scolarisé ; Nprim : niveau primaire ; Nsecond : niveau secondaire ; Diva : liberté ; $\mathrm{MoCl}$ : mode claustration ; Rameli : race améliorée ; Cvccalm : critère de choix verrat calme ; Cvcagres : critère de choix verrat agressif ; Cvtesmde : critère de choix testicules moyennement développés : Cvtesde : critère de choix testicules développés ; Cvtesbde : critère de choix testicules bien développés; Cvcroi : critère de choix verrat croissance : Cvssain : critère de choix verrat sain ; Cvconf : critère de choix verrat conformation; Cvgenet : critère de choix verrat absence de défaut génétique Cvtétine : critère de choix verrat nombre de tétines ; RvVei : critère de réforme verrat devient vieux ; Rvagres : critère de réforme verrat devient agressif ; Rvlibi : critère de réforme perte de libido.
La taille du cheptel était plus grande $(\mathrm{p}<0,05)$ dans le groupe 2 que dans le groupe 3 , et plus grande dans le groupe 3 que dans le groupe 1 pour les critères de choix et de réforme des mâles (tableau III). Le nombre de femelles dépendait du type d'élevage et il était plus faible dans le groupe 1 que dans les deux autres groupes pour les critères de choix et de réforme des deux sexes (tableau III). Le nombre moyen de truies de tous les élevages a été de 3,3. Ce nombre a été plus élevé dans le groupe 2 et plus bas dans le groupe 1 pour les critères de choix et de réforme des mâles (tableau III). Les nombres de porcelets, de jeunes mâles, ou de jeunes cochettes n'ont pas varié significativement d'un groupe à l'autre.

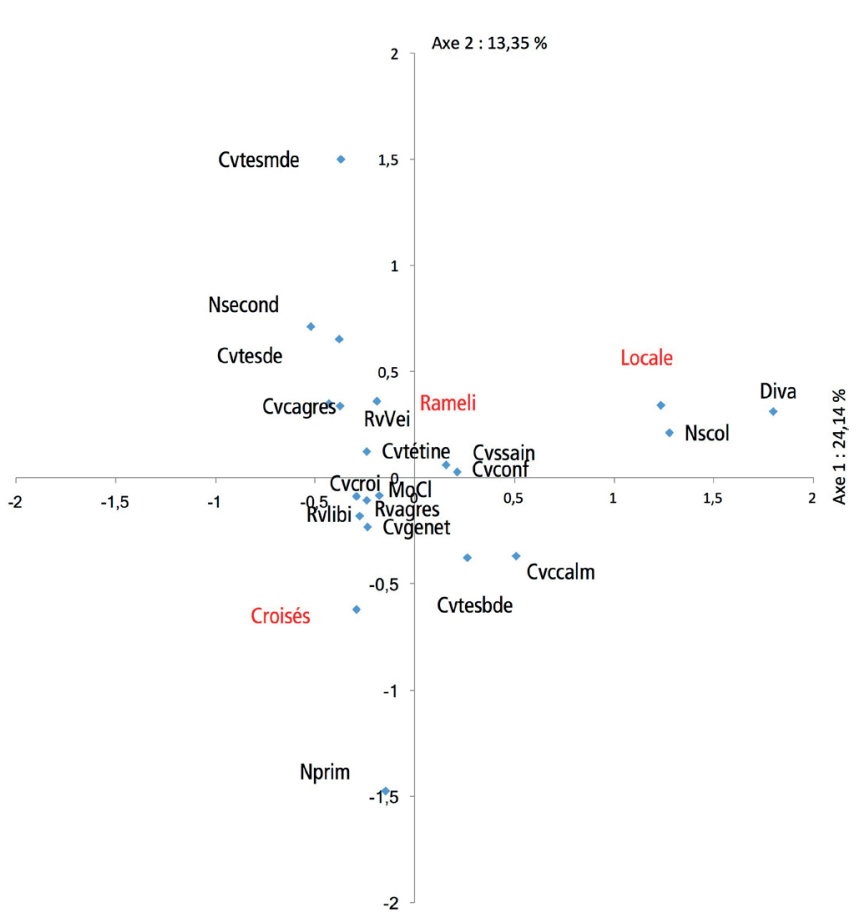




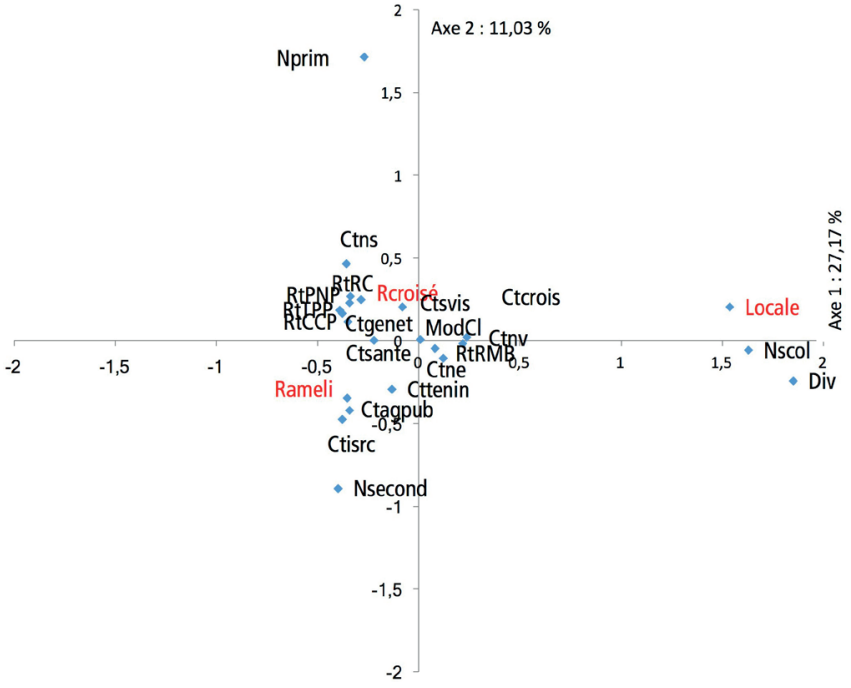

Figure 3: critères de choix et de réforme des femelles dans les départements de l'Ouémé et du Plateau au Sud-Bénin. Nscol non scolarisé ; Nprim : Niveau primaire; Nsecond : niveau secondaire ; Diva : liberté ; $\mathrm{MoCl}$ : mode claustration ; Rameli : race améliorée ; Rcroisé : race croisée ; Ctns : critère de choix truie nombre de sevrés ; Ctne : critère de choix nombre de nés ; Ctnv : critère de choix nombre de nés vivants ; Ctcroi : critère de choix truie croissance; Ctsante : critère de choix truie saine ; Ctagpub : critère de choix truie âge à la puberté ; Ctgenet : critère de choix truie absence de défaut génétique; Ctisrc : critère de choix truie intervalle sevrage retour des chaleurs; Ctsvis : critère de choix truie survie au sevrage; Cttenin : critère de choix truie nombre de tétines; RtRMB : critères de réforme truie rang de mise bas ; RtTPP : critère de réforme truie taille de la portée devient petite ; RtPNP : critère de réforme truie poids des porcelets à la naissance devient petit ; RtCCP : critères de réforme truie croissance des porcelets devient lente; RtRC : critère de réforme truie retour plus rapide des chaleurs après sevrage.

\section{Tableau III}

Structure du cheptel porcin par groupe d'éleveurs d'après les critères de choix et de réforme des reproducteurs mâles et des femelles au Sud-Bénin

\begin{tabular}{|c|c|c|c|c|c|c|c|c|c|c|c|c|}
\hline \multirow[t]{2}{*}{ Variables } & \multicolumn{6}{|c|}{ D'après les critères de choix et de réforme des mâles } & \multicolumn{6}{|c|}{ D'après les critères de choix et de réforme des femelles } \\
\hline & $\begin{array}{l}\text { Moyenne } \\
\text { générale } \\
(n=65)\end{array}$ & $\begin{array}{c}\text { Groupe } 1 \\
(n=11)\end{array}$ & $\begin{array}{c}\text { Groupe } 2 \\
(n=27)\end{array}$ & $\begin{array}{c}\text { Groupe } 3 \\
(n=27)\end{array}$ & DSR & $\begin{array}{l}\text { Ecart entre } \\
\text { groupes (test } \\
\text { de Fisher) }\end{array}$ & $\begin{array}{l}\text { Moyenne } \\
\text { générale } \\
(n=62)\end{array}$ & $\begin{array}{c}\text { Groupe } 1 \\
(n=13)\end{array}$ & $\begin{array}{c}\text { Groupe } 2 \\
(n=26)\end{array}$ & $\begin{array}{c}\text { Groupe } 3 \\
(n=23)\end{array}$ & DSR & $\begin{array}{l}\text { Ecart entre } \\
\text { groupes (test } \\
\text { de Fisher) }\end{array}$ \\
\hline Verrat & 1,2 & $0,7^{b}$ & $1,3^{\mathrm{a}}$ & 1,3 & 1,1 & * & 1,1 & $0,5^{b}$ & $1,2^{a, b}$ & $1,4^{\mathrm{a}}$ & 1 & * \\
\hline Truie & 3,3 & $1,9^{b}$ & $4,3^{\mathrm{a}}$ & $3^{a, b}$ & 3,9 & $* *$ & 3,3 & $1,5^{\mathrm{a}}$ & $3,8^{\mathrm{a}}$ & $3,7^{\mathrm{a}}$ & 3,9 & NS \\
\hline $\begin{array}{l}\text { Porcelet mâle } \\
\text { non sevré }\end{array}$ & 1,6 & $1,5^{\mathrm{a}}$ & $1,3^{\mathrm{a}}$ & $2,1^{\mathrm{a}}$ & 4,4 & NS & 1,7 & $1,8^{\mathrm{a}}$ & $1,7^{\mathrm{a}}$ & $1,7^{\mathrm{a}}$ & 4,5 & NS \\
\hline $\begin{array}{l}\text { Porcelet femelle } \\
\text { non sevré }\end{array}$ & 2 & $1,7^{\mathrm{a}}$ & $1,8^{\mathrm{a}}$ & $2,4^{\mathrm{a}}$ & 5,7 & NS & 2,1 & $1,8^{\mathrm{a}}$ & $2,3^{\mathrm{a}}$ & $2^{\mathrm{a}}$ & 5,9 & NS \\
\hline Jeune mâle entier & 3,2 & $1,4^{\mathrm{a}}$ & $3,4^{\mathrm{a}}$ & $3,9^{\mathrm{a}}$ & 5,9 & NS & 3,2 & $1,2^{\mathrm{a}}$ & $3^{a}$ & $4,6^{\mathrm{a}}$ & 6,1 & NS \\
\hline Jeune mâle castré & é 3 & $1,6^{\mathrm{a}}$ & $4,1^{\mathrm{a}}$ & $2,3^{\mathrm{a}}$ & 6,8 & NS & 3,1 & $1^{\mathrm{a}}$ & $5,3^{\mathrm{a}}$ & $1,9^{\mathrm{a}}$ & 6,7 & NS \\
\hline Cochette & 4,6 & $1,2^{\mathrm{a}}$ & $6,7^{\mathrm{a}}$ & $3,8^{\mathrm{a}}$ & 10 & NS & 4,5 & $0,7^{\mathrm{a}}$ & $6,2^{\mathrm{a}}$ & $4,8^{\mathrm{a}}$ & 10,3 & NS \\
\hline Effectif total & 18,9 & $10,1^{\mathrm{c}}$ & $22,9^{a}$ & $18,6^{b}$ & 26,4 & $* * *$ & 19,1 & $8,3^{\mathrm{a}}$ & $23,4^{\mathrm{a}}$ & $20,2^{a}$ & 26,8 & NS \\
\hline
\end{tabular}

${ }^{*} p<0,05 ;{ }^{* *} p<0,01 ;{ }^{* *} p<0,001 ;$ DSR : déviation standard résiduelle ; NS : non significatif

$\mathrm{a}, \mathrm{b}$ Les moyennes intraclasses sur une même ligne suivies de la même lettre ne diffèrent pas significativement au seuil de $5 \%$.

\section{Critères de choix des reproducteurs}

Les critères les plus utilisés dans les 65 élevages pour le choix des verrats ont été l'état de santé (89,2\%), la conformation (80\%), la croissance $(69,2 \%)$, l'absence de défauts génétiques $(66,15 \%)$, les testicules très développés $(58,5 \%)$ et le nombre de tétines $(50,8 \%)$. La proportion d'éleveurs qui utilisaient les critères conformation, état de santé, nombre de tétines et croissance du porcelet entre la naissance et le sevrage n'a pas différé significativement d'un groupe à l'autre. En revanche, l'absence de défauts génétiques et le caractère agressif ont été davantage utilisés $(\mathrm{p}<0,05)$ dans les groupes 2 et 3 que dans le groupe 1. Les défauts génétiques recherchés par ces éleveurs étaient la dépression au niveau du dos, le défaut de conformation, les problèmes d'aplomb, le défaut de taille et les diverses malformations. Les verrats sélectionnés devaient avoir un bon aplomb et un bon développement musculaire. L'âge à l'entrée en reproduction des verrats choisis n'a pas varié significativement d'un groupe à l'autre. Il a été en moyenne de 7,9 mois avec un minimum de 5 mois et un maximum de 12 . Le nombre moyen des tétines des verrats sélectionnés a été de $12,5(\mathrm{n}=32)$ avec un minimum de 6 tétines et un maximum de 16. Après la sélection, le reste des mâles étaient castrés à l'âge de 81 jours avec un âge minimum à la castration de 14 jours et maximum de 150 .

Les critères les plus utilisés pour le choix des femelles dans tous les élevages enquêtés ont été l'état de santé ( $82,3 \%)$, les liens de parenté $(72,6 \%)$, la croissance entre la naissance et le sevrage $(75,8 \%)$, la taille de la portée de naissance $(66,1 \%)$, le nombre de nés vivants dans la portée de naissance $(59,7 \%)$, le nombre de tétines des cochettes $(58,1 \%)$ et l'absence de défauts génétiques (51,6\%) (tableau IV). Le nombre de tétines, l'absence de défauts génétiques et les liens de parenté ont été davantage utilisés dans les groupes 2 et 3 que dans le groupe 1. Les critères les moins utilisés ont été l'âge à la puberté, le nombre de porcelets sevrés dans la portée d'origine, l'aptitude maternelle et l'intervalle sevrage-œstrus. Les défauts génétiques recherchés ont été les mêmes que ceux utilisés pour le choix des verrats. L'intervalle sevrage-saillie a été utilisé par certains (33,9\%) pour maintenir ou non la femelle sélectionnée après la première mise bas. Ce critère n'a pas été utilisé dans le groupe 1 mais il l'a été par 38,5\% des éleveurs du groupe 2 et $47,8 \%$ des éleveurs du groupe 3. L'âge moyen d'entrée en reproduction des cochettes de tous les élevages a 
été de 7,8 mois avec un âge minimum de 5 mois et maximum de 12 . La taille moyenne de la portée de naissance des cochettes de tous les élevages a été de 8,9 porcelets avec une taille minimum de 5 porcelets et maximum de 12. Cette taille était plus élevée $(p<0,001)$ dans les groupes 2 et 3 que dans le groupe 1 (tableau V). L'intervalle moyen sevrage-saillie dans tous les élevages a été de 36,4 jours avec un minimum de 4 jours et un maximum de 90 . Cet intervalle n'a pas varié significativement d'un groupe à l'autre. Le nombre moyen de tétines des cochettes a été de $12,8(\mathrm{n}=32)$.

\section{Critères de réforme des reproducteurs}

Les critères de réforme des verrats dans tous les élevages ont été la perte de libido (66,1\%), l'âge $(61,5 \%)$ et le changement de caractère (44,6 \%). La proportion d'éleveurs qui utilisaient ces critères n'a pas varié significativement d'un groupe à l'autre, mais ces critères ont été utilisés par la majorité des éleveurs des groupes 2 et 3 (tableau VI). L'âge moyen à la réforme des verrats a été de 2,2 ans avec un âge minimum de 1 an et maximum de 5 .

Les critères de réforme des truies dans tous les élevages ont été le rang de portée (79\%), la baisse de la taille de la portée $(53,2 \%)$, la baisse de poids à la naissance $(45,2 \%)$, la baisse de croissance des porcelets sous la mère $(33,9 \%)$ et les chaleurs tardives après le sevrage $(37,1 \%)$. Les éleveurs ont davantage utilisé $(p<0,05)$ le critère baisse de la portée dans les groupes 2 et 3 que dans le groupe 1 (tableau VI). La proportion d'éleveurs qui utilisaient les critères baisse de poids de naissance des porcelets, baisse de croissance des porcelets et chaleurs tardives après le sevrage n'a pas différé significativement d'un groupe à l'autre. La taille moyenne de la portée à la réforme a été de 5,7 porcelets avec une taille minimum de 5 porcelets et maximum de 6 dans tous les élevages, et n'a pas varié d'un groupe

\section{Tableau IV}

Critères de choix des reproducteurs porcins au Sud-Bénin

\begin{tabular}{|c|c|c|c|c|c|c|c|c|}
\hline \multirow[t]{2}{*}{ Variables } & \multirow[t]{2}{*}{$\%$ général } & \multicolumn{2}{|c|}{ Groupe 1} & \multicolumn{2}{|c|}{ Groupe 2} & \multicolumn{2}{|c|}{ Groupe 3} & \multirow{2}{*}{$\begin{array}{l}\text { Ecart entre groupes } \\
\text { (test du } \chi^{2} \text { ) }\end{array}$} \\
\hline & & $\%$ & IC & $\%$ & IC & $\%$ & IC & \\
\hline \multicolumn{9}{|l|}{ Critères de choix des verrats } \\
\hline Effectif & 65 & 11 & & 27 & & 27 & & - \\
\hline Conformation & 80,0 & $90,9^{\mathrm{a}}$ & {$[73,9-107,9]$} & $85,2^{\mathrm{a}}$ & {$[71,8-98,6]$} & $70,4^{\mathrm{a}}$ & {$[53,2-87,6]$} & NS \\
\hline Etat de santé & 89,2 & $100^{\mathrm{a}}$ & [100-100] & $85,2^{\mathrm{a}}$ & {$[71,8-98,6]$} & $88,9^{a}$ & {$[77,1-100,7]$} & NS \\
\hline $\begin{array}{l}\text { Absence de défaut } \\
\text { génétique }\end{array}$ & 66,2 & $36,4^{b}$ & [8-64,8] & $66,7^{a}$ & {$[48,9-84,5]$} & $77,8^{\mathrm{a}}$ & {$[62,1-93,5]$} & $*$ \\
\hline Caractère calme & 46,1 & $54,5^{\mathrm{a}}$ & {$[25,1-83,9]$} & $40,7^{\mathrm{a}}$ & {$[22,2-59,2]$} & $48,1^{\mathrm{a}}$ & {$[29,3-66,9]$} & NS \\
\hline Caractère agressif & 43,01 & $18,2^{b}$ & {$[-4,6-41]$} & $51,8^{\mathrm{a}}$ & {$[33-70,6]$} & $44,4^{\mathrm{a}}$ & {$[25,7-63,1]$} & $*$ \\
\hline $\begin{array}{l}\text { Testicules moyennement } \\
\text { développés }\end{array}$ & 6,1 & $0,0^{a, b}$ & [0-0] & $14,8^{\mathrm{a}}$ & {$[1,4-28,2]$} & $0,0^{b}$ & [0-0] & * \\
\hline Testicules développés & 18,5 & $0,0^{\mathrm{a}}$ & {$[0-0]$} & $25,9^{a}$ & {$[9,4-42,4]$} & $18,5^{\mathrm{a}}$ & {$[3,9-33,1]$} & NS \\
\hline Testicules très développés & 58,5 & $90,9^{a}$ & {$[73,9-107,9]$} & $44,4^{b}$ & {$[25,7-63,1]$} & $59,3^{\mathrm{a}}$ & {$[40,8-77,8]$} & $*$ \\
\hline $\mathrm{Nb}$. de tétines & 50,8 & $36,4^{\mathrm{a}}$ & {$[8-64,8]$} & $51,8^{\mathrm{a}}$ & {$[33-70,6]$} & $55,6^{\mathrm{a}}$ & {$[36,9-74,3]$} & NS \\
\hline $\begin{array}{l}\text { Croissance entre } \\
\text { naissance et sevrage }\end{array}$ & 69,23 & $54,5^{\mathrm{a}}$ & {$[25,1-83,9]$} & $81,5^{\mathrm{a}}$ & {$[66,9-96,1]$} & $63,0 \mathrm{a}$ & {$[44,8-81,2]$} & NS \\
\hline Liens de parenté & 40,0 & $0,0^{b}$ & {$[0-0]$} & $44,4^{\mathrm{a}}$ & {$[25,7-63,1]$} & $51,8^{\mathrm{a}}$ & {$[33-70,6]$} & * \\
\hline \multicolumn{9}{|c|}{ Critères de choix des cochettes } \\
\hline Effectif & 62 & 13 & & 26 & & 23 & & - \\
\hline Age à la puberté & 38,7 & $15,4^{\mathrm{a}}$ & {$[-4,2-35,0]$} & $46,1^{\mathrm{a}}$ & {$[26,9-65,3]$} & $43,5^{\mathrm{a}}$ & {$[23,2-63,8]$} & NS \\
\hline $\begin{array}{l}\text { Nés totaux de } \\
\text { la portée d'origine }\end{array}$ & 66,1 & $61,5^{\mathrm{a}}$ & {$[35,0-88,0]$} & $76,9^{\mathrm{a}}$ & {$[60,7-93,1]$} & $56,5^{\mathrm{a}}$ & {$[36,2-76,8]$} & NS \\
\hline Nés vivants & 59,7 & $53,8^{\mathrm{a}}$ & {$[26,7-80,9]$} & $61,5^{\mathrm{a}}$ & {$[42,8-80,2]$} & $60,9^{a}$ & {$[41,0-80,8]$} & NS \\
\hline Nb. de sevrés & 24,2 & $7,7^{\mathrm{a}}$ & {$[-6,8-22,2]$} & $23,1^{\mathrm{a}}$ & {$[6,9-39,3]$} & $34,8^{a}$ & {$[15,3-54,3]$} & NS \\
\hline Aptitude maternelle & 27,4 & $15,4^{\mathrm{a}}$ & {$[-4,2-35,0]$} & $23,1^{\mathrm{a}}$ & {$[6,9-39,3]$} & $39,1^{\mathrm{a}}$ & {$[19,2-59,0]$} & NS \\
\hline $\begin{array}{l}\text { Croissance entre } \\
\text { naissance et sevrage }\end{array}$ & 75,8 & $61,5^{\mathrm{a}}$ & {$[35,0-88,0]$} & $88,5^{a}$ & {$[76,2-100,8]$} & $69,6^{a}$ & {$[50,8-88,4]$} & NS \\
\hline $\begin{array}{l}\text { Intervalle sevrage-œestrus } \\
\text { de la } 1^{\text {re }} \text { portée }\end{array}$ & 33,9 & $0^{b}$ & {$[0,0-0,0]$} & $38,5^{\mathrm{a}}$ & {$[19,8-57,2]$} & $47,8^{\mathrm{a}}$ & {$[27,4-68,2]$} & $*$ \\
\hline $\begin{array}{l}\mathrm{Nb} \text {. de tétines } \\
\text { des cochettes }\end{array}$ & 58,1 & $23,1^{b}$ & {$[0,2-46,0]$} & $65,4^{\mathrm{a}}$ & {$[47,1-83,7]$} & $69,6^{\mathrm{a}}$ & {$[50,8-88,4]$} & $*$ \\
\hline $\begin{array}{l}\text { Absence de } \\
\text { défaut génétique }\end{array}$ & 51,6 & $15,4^{b}$ & {$[-4,2-35,0]$} & $61,5^{\mathrm{a}}$ & {$[42,8-80,2]$} & $60,9^{a}$ & {$[41,0-80,8]$} & * \\
\hline $\begin{array}{l}\text { Etat de santé de } \\
\text { la cochette }\end{array}$ & 82,3 & $84,6^{\mathrm{a}}$ & {$[65,0-104,2]$} & $80,8^{\mathrm{a}}$ & {$[65,7-95,9]$} & $82,6^{\mathrm{a}}$ & {$[67,1-98,1]$} & NS \\
\hline Lien de parenté & 72,6 & $30,8^{b}$ & {$[5,7-55,9]$} & $88,5^{a}$ & {$[76,2-100,8]$} & $78,3^{\mathrm{a}}$ & {$[61,5-95,1]$} & $* * *$ \\
\hline
\end{tabular}

${ }^{*} \mathrm{p}<0,05 ;{ }^{* *} \mathrm{p}<0,01 ;{ }^{* * *} \mathrm{p}<0,001 ; \mathrm{NS}$ : non significatif; IC : intervalle de confiance

$a, b$ Les pourcentages sur une même ligne suivis de la même lettre ne diffèrent pas significativement au seuil de $5 \%$. 
Tableau V

Effet de la catégorie d'éleveurs sur les moyennes de l'âge à l'entrée en reproduction, au sevrage, à la réforme et à la castration, la taille de portée, le rang de portée et la durée de l'intervalle sevrage-saillie des porcs au Sud-Bénin

\begin{tabular}{|c|c|c|c|c|c|c|c|c|c|c|}
\hline \multirow[t]{2}{*}{ Variables } & \multicolumn{2}{|c|}{ Moy. générale } & \multicolumn{2}{|c|}{ Groupe 1} & \multicolumn{2}{|c|}{ Groupe 2} & \multicolumn{2}{|c|}{ Groupe 3} & \multirow[t]{2}{*}{ DSR } & \multirow{2}{*}{$\begin{array}{l}\text { Ecart entre } \\
\text { groupes (test } \\
\text { de Fisher) }\end{array}$} \\
\hline & $\mathbf{N}$ & Moy. & $\mathbf{N}$ & Moy. & $\mathbf{N}$ & Moy. & $\mathbf{N}$ & Moy. & & \\
\hline \multicolumn{11}{|c|}{ Suivant les critères de choix et de réforme des mâles } \\
\hline $\begin{array}{l}\text { Age d'entrée en reproduction } \\
\text { cochette (mois) }\end{array}$ & 46 & 7,9 & 3 & $9,3^{a}$ & 25 & $7,8^{b}$ & 18 & $7,8^{b}$ & 1,5 & * \\
\hline $\begin{array}{l}\text { Age d'entrée en reproduction } \\
\text { verrat (mois) }\end{array}$ & 39 & 7,9 & 3 & $7,7^{\mathrm{a}}$ & 20 & $8,3^{\mathrm{a}}$ & 16 & $7,4^{\mathrm{a}}$ & 1,4 & NS \\
\hline Age à la réforme de la truie (an) & 33 & 2,8 & 3 & $3,3^{\mathrm{a}}$ & 15 & $2,7^{\mathrm{a}}$ & 15 & $2,7^{\mathrm{a}}$ & 0,8 & NS \\
\hline Age à la réforme du verrat (an) & 22 & 2,2 & - & - & 14 & $2,1^{\mathrm{a}}$ & 8 & $2,2^{\mathrm{a}}$ & 0,8 & NS \\
\hline Age au sevrage (jours) & 47 & 54,1 & 3 & $54^{\mathrm{a}}$ & 24 & $56,1^{\mathrm{a}}$ & 20 & $51,6^{\mathrm{a}}$ & 17,9 & NS \\
\hline Age à la castration (jours) & 47 & 81,3 & 4 & $86,2^{\mathrm{a}}$ & 24 & $86^{\mathrm{a}}$ & 19 & $74,3^{\mathrm{a}}$ & 39,9 & NS \\
\hline Taille de portée de naissance & 44 & 9,3 & 7 & 6,1 & 18 & 10,2 & 19 & 9,7 & 0,9 & $* * *$ \\
\hline Taille de la portée à la réforme & 36 & 5,7 & 1 & 3 & 22 & 5,7 & 13 & 5,8 & 0,4 & $* * *$ \\
\hline Intervalle sevrage-saillie (jours) & 43 & 37,4 & 2 & $60^{\mathrm{a}}$ & 23 & $39,7^{b}$ & 18 & $31,9^{b}$ & 20,5 & $*$ \\
\hline Rang de portée & 54 & 4,3 & 10 & $5,2^{\mathrm{a}}$ & 24 & $4,1^{b}$ & 20 & $4,2^{b}$ & 1,1 & * \\
\hline $\begin{array}{l}\text { Nb. de tétines des verrats } \\
\text { sélectionnés }\end{array}$ & 32 & 12,5 & 4 & $11,5^{\mathrm{a}}$ & 13 & $12,8^{\mathrm{a}}$ & 15 & $12,5^{\mathrm{a}}$ & 2,5 & NS \\
\hline $\begin{array}{l}\text { Nb. de tétines des cochettes } \\
\text { sélectionnées }\end{array}$ & 39 & 12,8 & 4 & $12^{\mathrm{a}}$ & 13 & $13,5^{\mathrm{a}}$ & 19 & $12,4^{\mathrm{a}}$ & 2,4 & NS \\
\hline \multicolumn{11}{|c|}{ Suivant les critères de choix et de réforme des femelles } \\
\hline $\begin{array}{l}\text { Age d'entrée en reproduction } \\
\text { cochette (mois) }\end{array}$ & 44 & 7,8 & 1 & $7,0^{\mathrm{a}}$ & 23 & $7,8^{\mathrm{a}}$ & 20 & $7,9^{a}$ & 1,5 & NS \\
\hline $\begin{array}{l}\text { Age d'entrée en reproduction } \\
\text { verrat (mois) }\end{array}$ & 36 & 7,8 & 1 & 7,0 & 20 & 7,9 & 15 & 7,8 & 1,5 & NS \\
\hline Age à la réforme de la truie (an) & 30 & 2,8 & 2 & $3,5^{\mathrm{a}}$ & 16 & $2,9^{\mathrm{a}}$ & 12 & $2,6^{\mathrm{a}}$ & 0,8 & NS \\
\hline Age à la réforme du verrat (an) & 21 & 2,2 & 1 & 2,0 & 12 & 2,2 & 8 & 2,2 & 0,8 & NS \\
\hline Age au sevrage (jours) & 44 & 54,7 & 2 & 82 & 22 & 56,1 & 20 & 50,4 & 17,1 & $*$ \\
\hline Age à la castration (jours) & 44 & 80,0 & 5 & 117 & 23 & 84,3 & 16 & 62,4 & 36,3 & NS \\
\hline Taille de portée de naissance & 35 & 8,9 & 8 & $6,1^{b}$ & 16 & $9,9^{\mathrm{a}}$ & 11 & $9,6^{\mathrm{a}}$ & 0,9 & $* * *$ \\
\hline Taille de la portée à la réforme & 22 & 5,7 & 3 & $4,7^{\mathrm{a}}$ & 13 & $5,5^{\mathrm{a}}$ & 6 & $5,7^{\mathrm{a}}$ & 0,7 & NS \\
\hline Intervalle sevrage-saillie (jours) & 40 & 36,4 & 2 & $31,5^{\mathrm{a}}$ & 20 & $41,2^{\mathrm{a}}$ & 18 & $31,4^{\mathrm{a}}$ & 20,3 & NS \\
\hline Rang de portée & 48 & 4,4 & 11 & $5,2^{\mathrm{a}}$ & 20 & $4,1^{b}$ & 17 & $4,2^{b}$ & 1,1 & $*$ \\
\hline $\begin{array}{l}\text { Nb. de tétines des verrats } \\
\text { sélectionnés }\end{array}$ & 31 & 12,5 & 3 & 11,0 & 14 & 12,6 & 14 & 12,9 & 2,6 & NS \\
\hline $\begin{array}{l}\mathrm{Nb} \text {. de tétines des cochettes } \\
\text { sélectionnées }\end{array}$ & 37 & 12,8 & 3 & $12^{\mathrm{a}}$ & 20 & $12,7^{\mathrm{a}}$ & 17 & $13^{\mathrm{a}}$ & 2,5 & NS \\
\hline
\end{tabular}

* $p<0,05 ;{ }^{* *} p<0,01 ;{ }^{* * *} p<0,001$; Moy. : moyenne ; DSR : déviation standard résiduelle

$\mathrm{a}, \mathrm{b}$ Les moyennes sur une même ligne suivies de la même lettre ne diffèrent pas significativement au seuil de $5 \%$.

à l'autre. Le rang moyen de la portée à la réforme a été des 4,4 avec un minimum de 3 et un maximum de 8 . Ce rang a été beaucoup plus élevé $(\mathrm{p}<0,05)$ dans le groupe $1(5,2$ mises bas) que dans les groupes 2 (4,1 mises bas) et 3 (4,2 mises bas). L'âge à la réforme des truies n'a pas varié d'un groupe à l'autre et a été en moyenne de 2,8 ans $(n=30)$ avec un âge minimum de 1 an et maximum de 5.

\section{DISCUSSION}

\section{Profil des élevages enquêtés}

Les éleveurs du groupe 1 étaient en majorité non scolarisés et élevaient des porcs locaux. Ce groupe décrit bien l'élevage traditionnel signalé par la FAO au Burkina Faso (FAO, 2012). Cet élevage traditionnel des porcs est caractérisé par l'élevage en liberté des animaux et une claustration en période de culture et la nuit. Les habitats rencontrés dans ce mode d'élevage servent souvent de lieu de couchage pour les animaux et d'apport éventuel de compléments alimentaires (Agbokounou et al., 2016b). L'éleveur contribue peu à la gestion de l'habitat, de l'alimentation, de la reproduction et parfois de la santé (Agbokounou et al., 2016b). Un type d'élevage similaire a été décrit au Sud-Bénin (Youssao et al., 2008). Les mêmes auteurs ont décrit des types similaires aux groupes 2 et 3 , où les animaux étaient plus souvent élevés en claustration et où l'usage des races améliorées serait dû au niveau d'instruction des éleveurs qui cherchent à rentabiliser leurs exploitations. Les éleveurs des groupes 2 et 3 étaient en 


\section{Tableau VI}

Critères de réforme des reproducteurs porcins au Sud-Bénin

\begin{tabular}{|c|c|c|c|c|c|c|c|c|}
\hline \multirow[t]{2}{*}{ Variables } & \multirow[t]{2}{*}{ \% général } & \multicolumn{2}{|c|}{ Groupe 1} & \multicolumn{2}{|c|}{ Groupe 2} & \multicolumn{2}{|c|}{ Groupe 3} & \multirow{2}{*}{$\begin{array}{l}\text { Ecart entre groupes } \\
\left.\text { (test du } \chi^{2}\right)\end{array}$} \\
\hline & & $\%$ & IC & $\%$ & IC & $\%$ & IC & \\
\hline \multicolumn{9}{|l|}{ Critères de réforme des verrats } \\
\hline Effectif & 65 & 11 & & 27 & & 27 & & - \\
\hline Age (devient vieux) & 61,5 & $45,4^{\mathrm{a}}$ & {$[16-74,8]$} & $74,1^{\mathrm{a}}$ & {$[57,6-90,6]$} & $55,6^{\mathrm{a}}$ & {$[36,9-74,3]$} & NS \\
\hline $\begin{array}{l}\text { Changement de caractère } \\
\text { (dévient agressif) }\end{array}$ & 44,6 & $36,4^{\mathrm{a}}$ & {$[8-64,8]$} & $51,8^{\mathrm{a}}$ & {$[33-70,6]$} & $40,7^{\mathrm{a}}$ & {$[22,2-59,2]$} & NS \\
\hline Perte de libido & 66,1 & $45,4^{\mathrm{a}}$ & {$[16-74,8]$} & $77,8^{\mathrm{a}}$ & {$[62,1-93,5]$} & $63^{\mathrm{a}}$ & {$[44,8-81,2]$} & NS \\
\hline \multicolumn{9}{|l|}{ Critères de réforme des truies } \\
\hline Effectif & 62 & 13 & & 26 & & 23 & & - \\
\hline Rang de portée & 79 & $84,6^{\mathrm{a}}$ & {$[65,0-104,2]$} & $80,8^{a}$ & {$[65,7-95,9]$} & $73,9^{a}$ & {$[56,0-91,8]$} & NS \\
\hline $\begin{array}{l}\text { Baisse de la taille } \\
\text { de la portée }\end{array}$ & 53,2 & $23,1^{b}$ & {$[0,2-46,0]$} & $61,5^{\mathrm{a}}$ & {$[42,8-80,2]$} & $60,9^{a}$ & {$[41,0-80,8]$} & $*$ \\
\hline $\begin{array}{l}\text { Baisse du poids de } \\
\text { naissance des porcelets }\end{array}$ & 45,2 & $23,1^{\mathrm{a}}$ & {$[0,2-46,0]$} & $46,2^{\mathrm{a}}$ & {$[27,0-65,4]$} & $56,5^{\mathrm{a}}$ & {$[36,2-76,8]$} & NS \\
\hline $\begin{array}{l}\text { Baisse de croissance } \\
\text { des porcelets }\end{array}$ & 33,9 & $15,4^{\mathrm{a}}$ & {$[-4,2-35,0]$} & $26,9^{\mathrm{a}}$ & {$[9,9-43,9]$} & $52,2^{\mathrm{a}}$ & {$[31,8-72,6]$} & NS \\
\hline $\begin{array}{l}\text { Chaleurs tardives } \\
\text { après sevrage }\end{array}$ & 37,1 & $15,4^{\mathrm{a}}$ & {$[-4,2-35,0]$} & $46,1^{\mathrm{a}}$ & {$[26,9-65,3]$} & $39,1^{\text {a }}$ & {$[19,2-59,0]$} & NS \\
\hline
\end{tabular}

${ }^{*} \mathrm{p}<0,05 ; \mathrm{NS}$ : non significatif ; IC : intervalle de confiance

$a, b$ Les pourcentages sur une même ligne suivis de la même lettre ne diffèrent pas significativement au seuil de $5 \%$.

majorité des personnes scolarisées et de ce fait disposaient d'un meilleur niveau de technicité et de savoir-faire pour conduire l'élevage de ces races que les éleveurs du groupe 1 qui étaient en majorité non scolarisés et qui utilisaient des porcs locaux. Les porcs locaux sont des animaux rustiques qui supportent mieux les conditions d'élevage difficiles. La taille des exploitations montre qu'il n'y avait toujours pas d'éleveurs de porcs professionnels comme l'avait signalé Youssao et al. (2008) dans les exploitations porcines au Sud-Bénin.

\section{Critères de choix des reproducteurs}

La majorité des éleveurs utilisait les critères état de santé et croissance des porcelets entre la naissance et le sevrage pour le choix des verrats et des cochettes, en raison de leur préférence pour les animaux à croissance rapide et en bonne santé. Les pathologies les plus rencontrées par les éleveurs du Sud-Bénin sont la peste porcine africaine (PPA) et la gale (Youssao et al., 2018). Un animal malade ou fragile sur le plan sanitaire n'est pas aussi performant pour les accouplements, d'où les pertes des chaleurs. Le choix des animaux en bonne santé par les éleveurs permettrait de réduire ces pertes. La majorité des éleveurs du groupe 1 ignorait les critères importants comme l'absence de défauts génétiques et le nombre de tétines pour le choix des verrats et des cochettes. Cette ignorance serait due à leur niveau d'instruction et à la race utilisée pour le nombre de tétines puisque les truies locales utilisées mettaient souvent bas des portées de 7 à 8 porcelets en élevage amélioré (Youssao et al., 2009). Les éleveurs de ce groupe devraient cependant introduire ce critère, puisqu'ils pourraient avoir des portées supérieures, et l'absence de critère pose le problème d'allaitement pour insuffisance de tétines. De plus, très souvent les tétines présentes ne sont pas toutes fonctionnelles et le nombre de tétines est un caractère fortement héritable $\left(h^{2}=0,30\right)$ chez le porc (Canario, 2006). Outre le nombre de tétines, les éleveurs devraient faire attention à la qualité des tétines car l'héritabilité des fausses tétines est plus importante que celle des bonnes tétines (0,42 vs 0,39) (Canario, 2006).

Le niveau d'instruction justifiait également l'utilisation en majorité de l'intervalle sevrage-œstrus pour le maintien des cochettes sélectionnées dans les groupes 2 et 3 , car il y avait dans ces groupes davantage de personnes instruites que dans le groupe 1. Les éleveurs devraient être sensibilisés sur les effets des défauts génétiques sur la production car ces anomalies provoquent le plus souvent une dépréciation des carcasses à l'abattoir induisant des pertes économiques importantes (Riquet et al., 2011). Les liens de parenté utilisés pour le choix des verrats et des cochettes permettent aux éleveurs de réduire la consanguinité dans les élevages. Ce critère a été moins utilisé dans le groupe 1 à cause du mode d'élevage en plein air pratiqué dans ce groupe qui ne facilite pas le contrôle des liens filiaux. Les verrats agressifs ont été davantage utilisés dans les groupes 2 et 3 car les éleveurs du groupe 1 utilisaient des verrats locaux qui sont déjà plus agressifs que les verrats améliorés des autres groupes. Les éleveurs choisissent les verrats ayant des testicules bien développés puisque le volume de sperme produit par le verrat est proportionnel à la taille des testicules (Feller et al., 2004). Cependant, des études sur la qualité de la semence de ces verrats sont indispensables puisque la fertilité des verrats dépend surtout de la qualité du sperme et non du volume. Les critères taille de la portée de naissance et nombre de nés vivants utilisés lors du choix des cochettes permettaient aux éleveurs de disposer de femelles prolifiques. La majorité des éleveurs et surtout ceux du groupe 1 ignoraient les critères comme l'intervalle sevrageœstrus et l'âge à la puberté pour le choix des cochettes, ce qui serait dû au manque de suivi dans les élevages. L'intervalle sevrage-saillie était trop long dans les trois groupes et les éleveurs gagneraient en l'améliorant puisque l'impact économique est important compte tenu de l'augmentation du temps improductif. Cette situation pourrait s'expliquer par le manque de professionnalisme des éleveurs puisque, en général, les animaux devraient être sevrés plus tôt pour permettre 
le retour plus rapide des chaleurs. Ainsi, l'intervalle sevrage-saillie rapporté en zone tropicale est de 4,9 jours (Renaudeau et al., 2001). Lorsque les porcelets sont sevrés tôt (à moins de 35 jours d'âge), cela permet d'éviter des chaleurs de lactation qui sont responsables de décalage du moment de retour des chaleurs. Le rallongement de la durée de retour des chaleurs après le sevrage peut être dû à une mauvaise alimentation de la truie allaitante. Cette mauvaise alimentation oblige la truie à mobiliser les réserves de son organisme pour la production de lait pour les porcelets, ce qui entraîne une perte de son état qu'elle doit récupérer après le sevrage (IFIP, 2013).

\section{Critères de réforme des reproducteurs}

Les critères de réforme âge et perte de libido des mâles reproducteurs dans les trois groupes ont aussi été rapportés par Knecht et al. (2017) dans les centres d'insémination artificielle. D'autres critères comme la qualité médiocre de la semence, l'infertilité, les problèmes de locomotion, la faible valeur génétique et les maladies sont aussi utilisés pour la réforme des verrats en production porcine (Acosta et Rueda, 2009 ; Knox, 2016 ; Knecht et al., 2017). Le mode de saillie naturelle et le faible nombre de truies par exploitation limiteraient les problèmes de locomotion. Le troisième critère de réforme des verrats de cette étude (changement de caractère) serait dû au manque de dispositifs de sécurité pour l'éleveur lors de la réalisation des activités quotidiennes (entretien, distribution d'aliments, soins aux animaux). Les critères de réforme des verrats recensés ont été moins utilisés dans le groupe 1, même si la différence avec les autres groupes n'était pas significative. Ce constat montre que la plupart des éleveurs de ce groupe ne disposaient pas de critères de réforme des verrats. L'âge moyen à la réforme de 2,2 ans signalé par les éleveurs était précoce et ne permettait pas aux verrats d'exprimer toute leur potentialité. Plusieurs causes dont les maladies justifieraient la réforme des animaux à cet âge. Ce même âge est rapporté pour la réforme des verrats au Tchad (Logtene et Kabore-Zoungrana, 2010). Le critère perte de libido est lié à l'âge de l'animal car un animal vieux perd facilement la libido. Cependant, la monte constitue l'un des problèmes de réforme de jeunes verrats (Knox, 2016).

Les critères de réforme des truies les plus cités par les éleveurs, comme le rang de portée et la baisse de performance (baisse de la portée et du poids à la naissance), ont également été rapportés par Engblom et al. (2007) dans les exploitations porcines, mais les principales causes de réforme des truies sont la vieillesse et les troubles de la reproduction (Engblom et al., 2007 ; Mote et al., 2009 ; de Jong et al., 2014). La baisse du poids à la naissance des porcelets, utilisée surtout dans les groupes 2 et 3 comme critère de réforme, doit être couplée à la taille de la portée pour éviter de réformer les truies prolifiques ou de sélectionner des cochettes issues de petites portées. L'augmentation de la prolificité s'accompagne au fil du temps d'une diminution du poids moyen des porcelets et d'une augmentation de l'hétérogénéité entre les porcelets de la portée (Tribout et al., 2003 ; Quiniou, 2010). L'usage de ce critère doit être aussi amélioré puisque les éleveurs ne disposaient pas de poids de porcelet en dessous duquel la mère peut être réformée. Le critère poids de naissance devrait faire partir des critères de sélection parce qu'il permettrait d'avoir des sujets beaucoup plus homogènes à la naissance.

Les truies sont aussi réformées quand elles donnent des porcelets à croissance lente entre la naissance et le sevrage. Ce critère doit être utilisé avec beaucoup de prudence car il est influencé par plusieurs facteurs dont la température et l'alimentation de la mère (Renaudeau et al., 2001 ; Renaudeau et al., 2007). L'exposition à une forte température s'accompagne d'une diminution de la consommation d'aliment, d'une diminution de la vitesse de croissance des porcelets et augmente la perte de poids vif de la truie en lactation (Renaudeau et al., 2001 ; Renaudeau et al., 2007). Les rangs de portées signalés par les éleveurs des groupes 2 et 3 étaient similaires à celui de 4,4 rapporté par Engblom et al. (2007), et le rang de portée notifié dans le groupe 1 était similaire au rang de 4,9 rapporté par Zhao et al. (2015). Ce critère utilisé dans la majorité des élevages n'était pas un bon motif de réforme des truies, d'autant que les femelles étaient réformées à 2,2 ans. Par ailleurs, le nombre de truies réformées diminue lorsque le rang de portée augmente jusqu'au sixième rang de portée, et l'augmentation du rang de portée améliore la taille de la portée et réduit le temps improductif dans l'élevage (Engblom et al., 2007). Les raisons de la réforme précoce des cochettes sont les troubles de reproduction et de locomotion (Engblom et al., 2007 ; de Hollander et al., 2015 ; Zhao et al., 2015). Les troubles de reproduction qui entraînent la réforme des cochettes sont l'anœstrus, les écoulements vulvaires, les avortements, les échecs de saillies, les dystocies, les momifications et les mort-nés (Tummaruk et al., 2009 ; de Jong et al., 2014 ; de Hollander et al., 2015 ; Zhao et al., 2015). La réforme précoce à l'âge de deux ans ou après la troisième mise bas peut entraîner des pertes à l'éleveur parce que les truies n'expriment pas tout leur potentiel avant cette période, la taille de la portée s'améliorant quand le rang de mise bas augmente (Logtene et Kabore-Zoungrana, 2010). Selon Buldgen et al. (1994), la réforme des reproductrices dans les élevages traditionnels telle qu'elle était pratiquée dans le groupe 1 est rarement basée sur des critères objectifs comme l'hypoprolificité, le cannibalisme ou l'agalactie. Le taux d'avortements a été signalé comme critère de réforme des truies au Tchad (Logtene et Kabore-Zoungrana, 2009) alors qu'aucun éleveur ne l'a signalé dans cette étude. Cela pourrait s'expliquer par la rareté des maladies abortives dans la zone et le manque d'enregistrement des performances dans les élevages.

\section{CONCLUSION}

L'étude sur les critères de choix et de réforme des reproducteurs a permis de décrire trois groupes d'éleveurs pour les verrats, et trois groupes pour les cochettes et les truies. Le groupe 1 était composé des personnes non scolarisées élevant des porcs locaux en liberté. Le groupe 2 comportait davantage d'éleveurs scolarisés qui élevaient des porcs améliorés et des porcs croisés en claustration. Le groupe 3 comprenait principalement des personnes non scolarisées ou ayant le niveau du secondaire utilisant des porcs améliorés et croisés en claustration. Les éleveurs des groupes 2 et 3 utilisaient des critères pertinents pour le choix des reproducteurs. Il faudrait améliorer les pratiques dans les élevages traditionnels où les éleveurs ignorent des critères importants comme les défauts génétiques, le nombre de tétines et la taille des portées sevrées. Il en est de même pour les critères de réforme car très peu d'éleveurs disposaient de critères de réforme des verrats.

\section{Remerciements}

Les auteurs remercient l'Académie de recherche et d'enseignement supérieur (ARES) de la Commission de la coopération au développement $(\mathrm{CCD})$ de la Belgique pour son appui à la réalisation de ces travaux à travers le projet de recherche-développement intitulé «Professionnalisation et renforcement de la compétitivité de la filière porc par la recherche-action en partenariat dans les départements de l'Ouémé et du Plateau au sud-est du Bénin ».

\section{REFERENCES}

Accodji I Fiogbe E, Gangbazo K, 2009. Essai de valorisation d'Azolla (Azolla microphylla Kaulf) dans la production porcine en zone humide. Int. J. Biol. Chem. Sci., 3 (5): 890-898, doi : 10.4314/ijbcs.v3i5.51054.

Acosta M.J., Rueda M., 2009. A note on causes of boar removal in Cuban pig farms. Livest. Res. Rural Dev., 21 (4), www.Irrd.org/lrrd21/4/acos21052.htm

Agbokounou A.M., Ahounou G.S., Youssao A., Mensah G.A., Koutinhouin B., Hornick J.-L., 2016a. Ethnologie et potentialités du porc local d'Afrique. J. Anim. Plant Sci., 29 (3): 4665-4677 
Agbokounou M.A., Ahounou G.S., Youssao A.I.K., Mensah G.A., Koutinhouin B., Hornick J-L., 2016b. Caractéristiques de l'élevage du porc local d'Afrique. J. Anim. Plant Sci., 30 (1): 4701-4713

Buldgen A., Piraux M., Dieng A., Schmit G., Compère R., 1994. Les élevages de porcs traditionnels du bassin arachidier sénégalais. www.fao.org/ag/ AGa/agap/FRG/FEEDback/War/t4650b/t4650b0s.htm (consulté 25 avr. 2017

Canario L., 2006. Aspects génétiques de la mortalité des porcelets à la naissance et en allaitement précoce : relations avec les aptitudes maternelles des truies et la vitalité des porcelets. Thèse Doct. Génétique animale et comportement, Institut national agronomique Paris-Grignon (INAPG), France, 328 p.

Countrystat, 2017. Base de données statistiques. http://countrystat.org/home. aspx?c=BEN\&ta=053CPD050\&tr=7 (consulté 12 mai 2017)

De Hollander C.A., Knol E.F., Heuven H.C.M., Van Grevenhof E.M., 2015 Interval from last insemination to culling. II. Culling reasons from practice and the correlation with longevity. Livest. Sci., 181: 25-30, doi: 10.1016/j. livsci.2015.09.018

De Jong E., Appeltant R., Cools A., Beek J., Boyend F., Chiers K., Maes D., 2014. Slaughterhouse examination of culled sows in commercial pig herds. Livest. Sci., 167: 362-369, doi: 10.1016/j.livsci.2014.07.001

Djimènou D., Adoukonou-Sagbadja H., Koudande O.D., Chrysostome C.A.A.M., Hounzangbe-Adote S.M., Agbangla C., 2017. Caractéristiques sociodémographiques des éleveurs de porcs (Sus scrofa domesticus) et structure du cheptel porcin au sud du Bénin. Int. J. Biol. Chem. Sci. 11 (5): 2177-2193, doi : 10.4314/ijbcs.v11i5.19

Engblom L., Lundeheim N., Dalin A-M., Andersson K., 2007. Sow removal in Swedish commercial herds. Livest. Sci. 106 (1): 76-86, doi: 10.1016/j. livsci.2006.07.002

FAO, 2012. Secteur porcin Burkina Faso. FAO, Rome, Italie (Revues nationales de l'élevage de la division de la production et de la santé animales de la $\mathrm{FAO} ; 1)$

Feller D., Thilmant P., Wavreille J., Boudry C., 2004. Le verrat, la truie : Aspects techniques de la reproduction. Filière porcine wallonne, Gembloux, Belgique, 48 p.

Hedji C.C., Houinato M., Houndonougbo F., Fiogbe E., 2015 Assainissement de l'environnement par la valorisation des ressources non conventionnelles en alimentation de porcs en croissance. Int. J. Biol. Chem. Sci., 9 (4) : 1929-1936

IFIP, 2013. Mémento de l'éleveur du porc, 7e édn. IFIP, Paris, France, 364 p.

INSAE, 2015. Que retenir des effectifs de population en 2013 ? INSAE, Cotonou, Bénin, $33 \mathrm{p}$.

Knecht D., Jankowska-M akosa A., Duzinski K., 2017. Boar genotype as a factor shaping age-related changes in semen parameters and reproduction longevity simulations. Theriogenology 98: 50-56, doi: 10.1016/j. theriogenology.2017.04.050

Knox R.V., 2016. Artificial insemination in pigs today. Theriogenology, 85 83-93
Logtene Y.M., Kabore-Zoungrana C.Y., 2010. Dynamique des élevages et caractéristiques des producteurs de porcs de la ville de N’Djaména. In : Savanes africaines en développement : innover pour durer (éds SeinyBoukar L., Boumard P.), 20-23 avr. 2009, Garoua, Cameroun, 1-9

Mote B.E., Mabry J.W., Stalder K.J., Rothschild M.F., 2009. Evaluation of current reasons for removal of sows from commercial farms. Prof. Anim. Sci., 25 (1): 1-7, doi: 10.15232/S1080-7446(15)30672-0

Quiniou N., 2010. Hétérogénéité des porcelets issus de l'hyperprolificité. TechniPorc, 33 (2) : 19-26

Renaudeau D., Gourdine J.L., Anais C., 2007. Effet du niveau de température sur l'acclimatation à court et moyen terme du porc en croissance. Journ. Rech. Porcine, 39 : 69-76

Renaudeau D., Noblet J., Quiniou N., Dubois S., 2001. Influence de I'exposition au chaud et de la réduction du taux de protéines dans I'aliment sur les performances des truies multipares en lactation. Journ. Rech. Porcine, 33 : 181-187

Riquet J., Mercat M.J., lannuccelli N., Servin B., Pailhoux E., Larzul C., 2011. Recherche de causes génétiques des anomalies congénitales majeures chez le porc. In : $43^{\text {es }}$ Journ. recherche porcine, IFIP / INRA, Paris, France, 15-16 fév. 2011, 7-12

SAS, 2013. Base SAS 9.4 Procedures guide, statistical procedures, 2nd Edn. SAS Institute, Cary, NC, USA, 550 p.

Tribout T., Caritez J.-C., Gogué J., Gruant J., Billon Y., Bouffaud M., Lagant

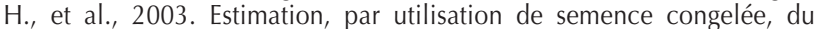
progrès génétique réalisé en France entre 1977 et 1998 dans la race porcine Large White : résultats pour quelques caractères de reproduction femelle. Journ. Rech. Porcine, 35 : 285-292

Tummaruk P., Kesdangsakonwut S., Kunavongkrit A., 2009. Relationships among specific reasons for culling, reproductive data, and gross morphology of the genital tracts in gilts culled due to reproductive failure in Thailand. Theriogenology, 71: 369-375, doi: 10.1016/j. theriogenology.2008.08.003

Youssao A.K.I., Dotché O.I., Seibou Toleba S., Kassa K.S., Assogba C., Ahounou S.G., Salifou C., Dahouda M., et al., 2018. Caractérisation phénotypique des ressources génétiques porcines des départements de l'Ouémé et du Plateau au Bénin. In : Journées scientifiques Quarantenaire de l'Ecole polytechnique d'Abomey-Calavi, 5-7 fév. 2018. Université d'Abomey-Calavi, Bénin, 77 p.

Youssao A.K.I., Koutinhouin G.B., Kpodekon T.M., Bonou A.G., Adjakpa A., Dotcho C.D.G., Atodjinou F.T.R., 2008. Pig production and indigenous genetic resources in suburban areas of Cotonou and Abomey-Calavi in Benin. Rev. Elev. Med. Vet. Pays Trop., 61 (3-4): 235-243, doi: 10.19182/ remvt.9995

Youssao A.K.I., Koutinhouin G.B., Kpodekon T.M., Bonou A.G., Adjakpa A., Ahounou G.S., Mourot J., 2009. Performances zootechniques et aptitudes bouchères des porcs locaux au Sud du Bénin. Bull. Anim. Health Prod. Afr., 57 (1) : 73-87

Zhao Y., Liu X., Mo D., Chen Q., Chen Y., 2015. Analysis of reasons for sow culling and seasonal effects on reproductive disorders in Southern China. Anim. Reprod. Sci., 159: 191-197, doi : 10.1016/j. anireprosci.2015.06.018 


\section{Summary}

Dotché I.O., Ahounou S.G., Salifou C.F.A., Biobou R., Kiki P.S., Govoeyi B., Antoine-Moussiaux N., Dehoux J.-P., Mensah G.A., Farougou S., Thilmant P., Youssao Abdou Karim I., Koutinhouin B. Selection and culling criteria for breeding boars and sows in pig farms from Oueme and Plateau departments in Benin

The aim of this study was to describe the selection and culling criteria for breeding pigs in Benin. A survey was carried out among 65 pig farms. The correspondence factor analysis was used to identify farmers' groups based on their practices at animal sex level. Group 1 consisted mostly of unschooled farmers mainly rearing free-range local-breed pigs. Group 2 farmers had primary or secondary education. Group 3 was composed of unschooled farmers or farmers with secondary education. The pig farmers of groups 2 and 3 used improved breeds and crossbreeds in confinement. The main criteria for male selection in farms were health status, conformation and highly developed testes, number of teats, absence of genetic defects and growth. The absence of genetic defects was more used in groups 2 and 3, and developed testes in group 1. The main criteria for sow selection in the three groups were health status, litter size, kinship and growth. Most of these criteria were less used in group 1. The most used criteria for boar culling were age and loss of libido. The main culling criteria for older sows in all groups were parity and decrease in litter size. The latter was more used in groups 2 and 3. To conclude, breeders had good criteria for breeding selection and reform that should be improved in order to increase productivity.

Keywords: swine, livestock management, boar, sow, reproductive performance, culling, Benin

\section{Resumen}

Dotché I.O., Ahounou S.G., Salifou C.F.A., Biobou R., Kiki P.S., Govoeyi B., Antoine-Moussiaux N., Dehoux J.-P., Mensah G.A., Farougou S., Thilmant P., Youssao Abdou Karim I., Koutinhouin B. Criterios de selección y sacrificio para verracos y cerdas reproductores en granjas porcinas de los departamentos de Oueme y Plateau en Benin

El objetivo de este estudio fue describir los criterios de selección y sacrificio para cerdos reproductores en Benin. Se realizó una encuesta entre 65 granjas porcinas. Se utilizó el análisis de los factores de correspondencia para identificar a los grupos de agricultores en base a sus prácticas a nivel de sexo animal. El grupo 1 consistió mayoritariamente de agricultores no escolarizados, criando principalmente cerdos de raza local de forma libre. Los agricultores del grupo 2 tenían educación primaria o secundaria. El grupo 3 estaba compuesto por agricultores sin educación o agricultores con educación secundaria. Los criadores de cerdos de los grupos 2 y 3 utilizaron cruces y razas mejoradas en estabulación. Los principales criterios para la selección de machos en las fincas fueron el estado de salud, la conformación y desarrollados importante de los testículos, el número de pezones, la ausencia de defectos genéticos y el crecimiento. La ausencia de defectos genéticos fue más utilizada en los grupos 2 y 3, luego desarrollo testicular en el grupo 1 . Los principales criterios para la selección de hembras en los tres grupos fueron el estado de salud, el tamaño de la camada, el parentesco y el crecimiento de lechones. La mayoría de estos criterios fueron menos utilizados en el grupo 1. Los criterios más utilizados para el sacrificio de verracos fueron la edad y la pérdida de la libido. Los principales criterios de sacrificio para las cerdas mayores en todos los grupos fueron la paridad y la disminución en el tamaño de la camada. Este último fue más utilizado en los grupos 2 y 3 . Para concluir, los criadores tenían buenos criterios para la selección y la modificación de reproducción que deberían mejorarse para aumentar la productividad.

Palabras clave: cerdo, manejo del ganado, verraco, cerda, reproductividad, eliminación, Benin 
